\title{
BMJ Open $\beta$-Adrenoreceptor agonists in the management of pain associated with renal colic: a systematic review
}

\author{
Andrew John Tabner, ${ }^{1}$ Graham David Johnson, ${ }^{1}$ Apostolos Fakis, ${ }^{2}$ Jane Surtees, ${ }^{2}$ \\ Robert lain Lennon ${ }^{1}$
}

To cite: Tabner AJ, Johnson GD, Fakis A, et al. $\beta$-Adrenoreceptor agonists in the management of pain associated with renal colic: a systematic review. BMJ Open 2016;6:e011315

doi:10.1136/bmjopen-2016011315

- Prepublication history for this paper is available online To view these files please visit the journal online (http://dx.doi.org/10.1136/ bmjopen-2016-011315)

Received 25 February 2016 Revised 5 May 2016 Accepted 16 May 2016

\section{(1) CrossMark}

\footnotetext{
${ }^{1}$ Emergency Department, Royal Derby Hospital, Derby, UK

${ }^{2}$ Research and Development Department, Derby Teaching Hospitals NHS Foundation Trust, Derby, UK
}

Correspondence to Dr Andrew John Tabner; andrew.tabner@nhs.net

\section{ABSTRACT}

Objectives: To determine whether $\beta$-adrenoreceptor agonists are effective analgesics for patients with renal colic through a systematic review of the literature.

Setting: Adult emergency departments or acute assessment units.

Participants: Human participants with proven or suspected renal colic.

Interventions: $\beta$-adrenoreceptor agonists.

Outcome measures: Primary. level of pain at $30 \mathrm{~min}$ following administration of the $\beta$-agonist. Secondary. level of pain at various time points following $\beta$-agonist administration; length of hospital stay; analgesic requirement; stone presence, size and position; degree of hydronephrosis.

Results: 256 records were screened and 4 identified for full-text review. No articles met the inclusion criteria.

Conclusions and implications: There is no evidence to support or refute the proposed use of $\beta$-agonists for analgesia in patients with renal colic. Given the biological plausibility and existing literature base, clinical trials investigating the use of $\beta$-adrenoreceptor agonists in the acute setting for treatment of the pain associated with renal colic are recommended.

Trial registration number: CRD42015016266.

\section{BACKGROUND}

\section{Description of the condition}

The lifetime incidence of renal calculi (kidney stones) is $\sim 12 \%$ in males and $6 \%$ in females $^{1}$ with a peak between 40 and 60 years of age. $^{2}$ Renal colic is the pain experienced by a patient when a renal calculus causes partial or complete obstruction of part of the renal outflow tract. Pain in renal colic is caused by ureteric spasm with increased peristalsis around the stone and dilation of the proximal urinary tract. Local irritation with prostaglandin release and inflammation further increases the renal pelvic pressure. ${ }^{3}$

\section{Strengths and limitations of this study}

- Full protocol published in advance of data collection on PROSPERO.

- Extensive searches including grey literature and handsearching.

- Only English language results (or those where a translation was available) were reviewed.

The clinical need that prompted this systematic review

Current analgesic regimes, usually involving a non-steroidal anti-inflammatory drug (NSAID) \pm an opiate, ${ }^{2}$ are often suboptimal; in some studies, less than half of the patients achieve complete pain relief within an acceptable time frame and a large proportion of patients require rescue analgesia within 4 hours. Additionally, side effects (especially vomiting) are relatively common with opioid analgesia. ${ }^{4}$

\section{Description of the intervention}

$\beta$-Adrenoreceptor agonists ( $\beta$-agonists) such as salbutamol/albuterol (a selective $\beta_{2}$-agonist ${ }^{5}$ in common use) are used as a treatment for acute exacerbations of asthma in both its nebulised and intravenous forms. The mechanism of action is via smooth muscle relaxation and subsequent reduction in bronchospasm. Salbutamol is an extensively used, wellestablished treatment with an established safety profile which is also licensed for tocolysis in premature labour. ${ }^{6}$

\section{How the intervention might work}

Various adrenoreceptor subtypes are present in the human ureter and their utility in the management of ureteral pathology is an area of scientific interest.

The $\alpha$-adrenoreceptors present in the ureter mediate ureteral contraction; ${ }^{7}$ their blockade reduces the time taken for ureteral 
stone passage, as well as reducing the frequency of pain episodes associated with renal colic. ${ }^{8}$

There is a body of laboratory evidence that $\beta_{2}$-adrenoreceptor and $\beta_{3}$-adrenoreceptor are expressed in the human ureter $^{9-12}$ and that their stimulation mediates ureteral relaxation. ${ }^{9-13}$ It has been demonstrated in animal models that intravenous isoproterenol results in a decrease in ureteral activity. ${ }^{14-17}$ Danuser $e t$ al ${ }^{18}$ demonstrated that both intravenous and topical isoproterenol reduced the frequency of ureteral contractions to $13 \%$ and $31 \%$ of controls, respectively. Recently, Jung et $a l^{19}$ achieved a reduction in renal pelvic pressure using endoluminal isoproterenol during ureterorenoscopic irrigation in human participants.

As yet, there is no direct evidence to inform the use of $\beta$-agonists as analgesic adjuncts in the treatment of pain associated with renal colic. It has been hypothesised ${ }^{20} 21$ that they may alleviate pain in patients with renal colic via ureteral relaxation mediated through $\beta$-adrenoreceptor stimulation. It is also worth noting that some $\beta$-agonists (eg, salbutamol) are at least partly excreted, unchanged, in the urine; ${ }^{22}$ as such, the potential for systemic and topical action exists.

\section{OBJECTIVES}

The primary objective of this systematic review was to determine whether $\beta$-agonists are effective analgesics for patients with renal colic. Secondary objectives include identifying any effect that $\beta$-agonists may have on length of hospital stay or alternative analgesic requirement. We also sought to determine any effect that the impact of stone presence, size and position, and the degree of hydronephrosis, may have on the efficacy of $\beta$-agonists as analgesics.

\section{Outcomes-primary}

Level of pain (assessed by any validated quantitative method) at $30 \mathrm{~min}$ following administration of the $\beta$-agonist.

\section{Outcomes-secondary}

A. Level of pain, assessed by any validated quantitative method-where possible data were to be extracted at $60 \mathrm{~min}, 120 \mathrm{~min}, 240 \mathrm{~min}, 8$ hours, 12 hours, 16 hours and 24 hours following administration of the $\beta$-agonist.

B. Length of stay in hospital (days).

C. Analgesic requirement, including medication used and cumulative dosage.

D. Stone presence, size and position as determined by radiological investigation.

E. Degree of hydronephrosis (expressed within any established grading system).

\section{METHODS}

Protocol and registration

The protocol for this systematic review has been prospectively registered on PROSPERO. It can be found at the following web address: http://www.crd.york.ac.uk/ PROSPERO/display_record.asp?ID=CRD42015016266\#. VVNR7tNVhBc

The protocol registration number is CRD42015016266.

\section{Study eligibility criteria}

We searched for randomised, quasi-randomised, casecontrol and cohort clinical trials that fulfilled the following criteria:

- Patients-human participants with either proven or suspected renal colic

- Interventions- $\beta$-adrenoreceptor agonists

- Comparator-any of: placebo; alternative analgesic regime

- Outcomes-any of: level of pain, assessed by any quantitative method; length of hospital stay; analgesic requirement; stone presence, size and position; degree of hydronephrosis

Lower level evidence such as case series or expert opinion pieces was not included within this systematic review. The background information discussed above provides adequate justification for considering the use of $\beta$-agonists in renal colic; as such, further expert opinion was not felt relevant to the study question. Case series alone would not provide adequate justification for changing practice and as such were excluded from the search results.

\section{Information sources}

The following databases were searched:

A. The Cochrane Database of Systematic Reviews (The Cochrane Library, issue 4, 2016)

B. MEDLINE (via OVID) (from January 1980 until 27 April 2016)

C. EMBASE (via OVID) (from January 1980 until 27 April 2016)

D. The NCBI PubMed database http://www.ncbi.nlm. nih.gov/pubmed—searched 27 April 2016

E. Centre for Reviews and Dissemination (CRD) http://www.crd.york.ac.uk/CRDWeb/ including the databases Database of Abstracts of Reviews of Effects (DARE), Health Technology Assessments (HTA) and NHS Economic Evaluation Database (NHS EED)

Note: At the time of searching and write-up, the following information was made available by DARE:

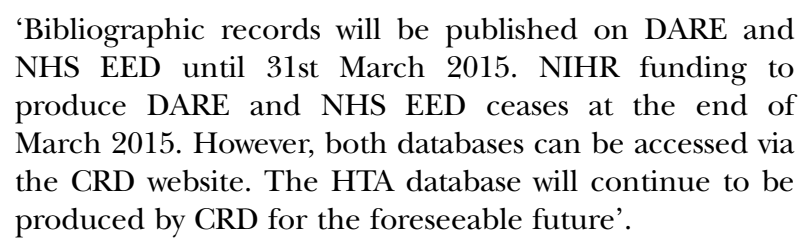

We restricted the results of our search to those involving human participants and those either written in English, with an English abstract or with an English translation provided (due to a lack of available funding for translation services). We did not restrict the date 
range of the results and incorporated both thesaurus headings and keyword searching.

References and conference abstracts were searched by hand and any suitable papers identified and reviewed.

We searched the following sources for existing guidelines that may have contained applicable information:

- National Institute for Health and Care Excellence (NICE)

- Turning Research into Practice (TRIP)

- National Guideline Clearinghouse

- Guidelines identified in the already-mentioned databases (MEDLINE, EMBASE, etc)

We searched the UK Clinical Trials Gateway ${ }^{23}$ and the National Institute for Health Research Clinical Research Network (NIHR CRN) Portfolio database ${ }^{24}$ for details of ongoing trials in the UK. Other ongoing trials will be searched via ClinicalTrials.gov, ${ }^{25}$ WHO International Clinical Trials Registry Platform (ICTRP) and Cochrane's CENTRAL database.

Any possible registered Systematic Reviews were searched for via PROSPERO (International Prospective Register of Systematic Reviews).

Relevant conference proceedings (Royal College of Emergency Medicine Annual Scientific Conference 2012-2014; European Society of Emergency Medicine European Congress 2012-2014; American College of Emergency Physicians Scientific Assembly 2012-2014; Japanese Urological Association Annual Meeting 2015; American Urological Association Annual Meeting 20122015) were also searched. A review of further conference proceedings was prevented by a lack of either professional organisation membership or online availability.

\section{Search strategy}

The following search strategy was used on MEDLINE; other database search strategies were comparable.

1. MEDLINE; exp RENAL COLIC/OR exp URINARY CALCULI/OR exp KIDNEY CALCULI/OR exp URETERAL OBSTRUCTION/; 39880 results.

2. MEDLINE; exp URETERAL CALCULI/; 5353 results.

3. MEDLINE; exp NEPHROLITHIASIS/; 15880 results.

4. MEDLINE; exp URETEROLITHIASIS/; 5429 results.

5. MEDLINE; (kidney AND stone OR 'kidney stone'). ti,ab; 3873 results.

6. MEDLINE; (ureteric AND colic OR 'ureteric colic'). ti,ab; 227 results.

7. MEDLINE; 1 OR 2 OR 3 OR 4 OR 5 OR 6; 41731 results.

8. MEDLINE; exp ALBUTEROL/; 8769 results.

9. MEDLINE; exp TERBUTALINE/; 2963 results.

10. MEDLINE; exp EPHEDRINE/; 4374 results.

11. MEDLINE; exp ISOPROTERENOL/; 28758 results.

12. MEDLINE; exp DOBUTAMINE/; 5665 results.

13. MEDLINE; exp XAMOTEROL/; 251 results.

14. MEDLINE; exp METAPROTERENOL/; 3160 results.

15. MEDLINE; exp CLENBUTEROL/; 1229 results.

16. MEDLINE; exp RITODRINE/; 916 results.
17. MEDLINE; (bambuterol OR formoterol OR indacaterol OR olodaterol OR salmeterol OR amibegron OR mirabegron OR solabegron OR denopamine OR bitolterol OR levosalbutamol OR ventolin OR ventmax OR salbutamol OR asmasal OR airomir OR 'air salb' OR salpin OR salamol).ti,ab; 9597 results.

18. MEDLINE; 8 OR 9 OR 10 OR 11 OR 12 OR 13 OR 14 OR 15 OR 16 OR 17; 56523 results.

19. MEDLINE; exp ADRENERGIC BETA-AGONISTS/; 103561 results.

20. MEDLINE; exp ADRENERGIC BETA-2 RECEPTOR AGONISTS/; 16296 results.

21. MEDLINE; 18 OR 19 OR 20; 109820 results.

22. MEDLINE; 7 AND 21; 43 results.

\section{Study selection}

Duplicates were removed using Zotero V.4.0. Citations were imported into Mendeley V.1.13.8 (which was used to manage references for the review); any further duplications reported at this stage were manually verified and merged.

Two investigators independently screened the title, abstract and keywords of every record identified by the search. If either investigator felt a paper merited further review, the full text of the article was examined.

All full-text articles were again reviewed independently by two investigators and assessed for eligibility against the above criteria. Differences in opinion were resolved by discussion; a third investigator would have mediated in the event that any discussions were unsuccessful.

\section{RESULTS}

The database searches identified 276 records; a further 13 records were found via alternative sources as detailed above. A total of 256 records remained after the removal of duplicates. Following the screening process, four articles were identified for full-text review: Aronson and Hauben; ${ }^{26}$ Erickson and Lieske; ${ }^{27}$ Helviz et $a l ;{ }^{28}$ and Reed. ${ }^{29}$ Details of the record management process during the review can be seen in figure 1 .

None of the articles reviewed at the full-text stage met any of the inclusion criteria for the systematic review. All had titles that did not allow immediate exclusion and three of the four papers did not have abstracts available for review; however, on full-text review, it was found that none of the above articles were clinical trials. Full details of reasons for exclusion can be seen in table 1 .

\section{DISCUSSION}

There is a disappointing lack of evidence to inform the use of $\beta$-agonists for analgesia in patients with renal colic; no relevant clinical trials were identified despite the broad inclusion criteria applied during the selection process.

There is a clear biological plausibility for the use of $\beta$-agonists in renal colic; the available evidence confirms both the presence of $\beta$-adrenoreceptors in the human 
Figure 1 PRISMA flow diagram demonstrating record handling during the systematic review.

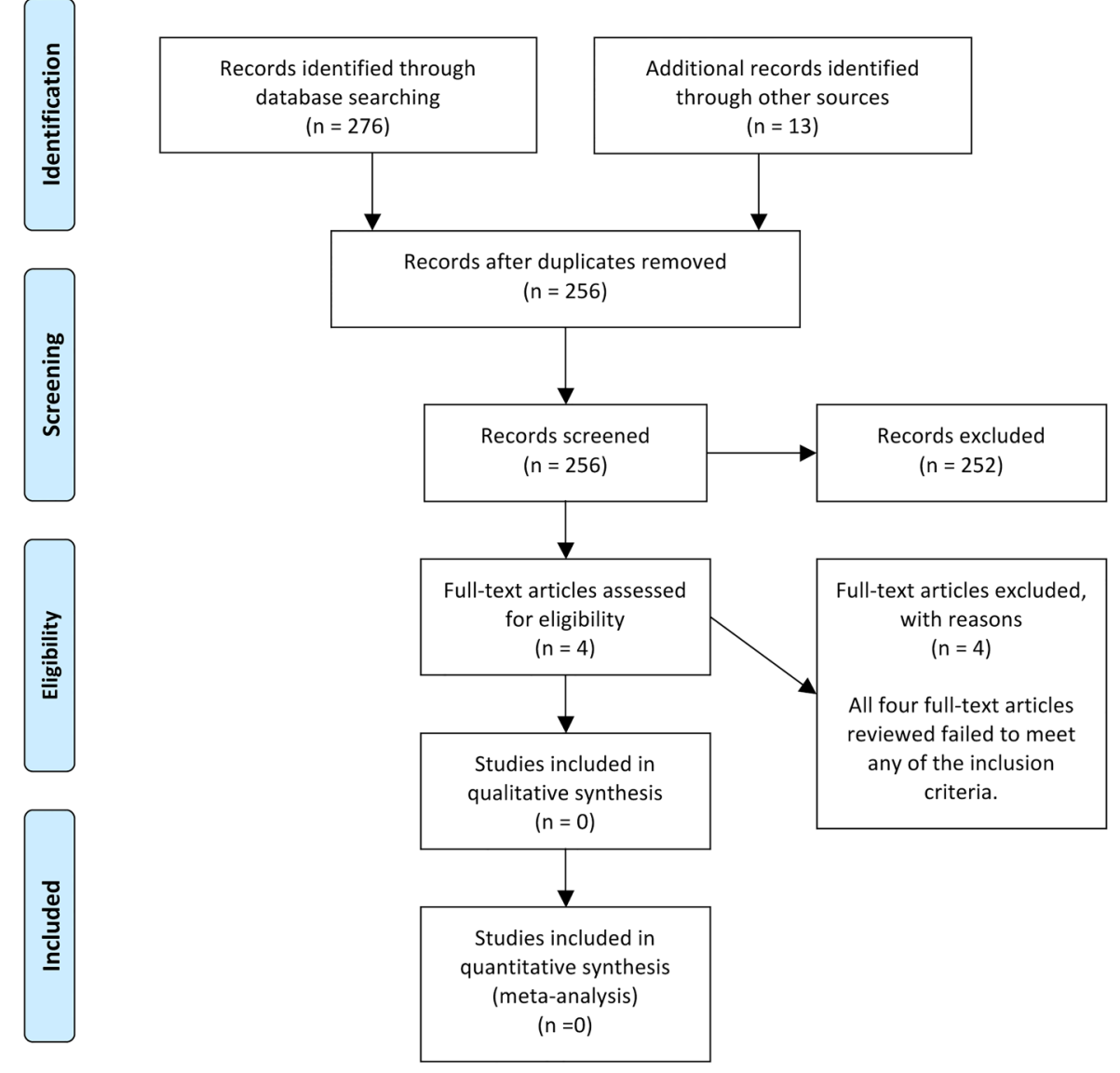

ureter and that their stimulation causes ureteral relaxation. This effect has been demonstrated in vivo, ${ }^{19}$ although in a different cohort of patients and for a different indication.

One potential explanation for the paucity of evidence on this topic is the recent research focus on the utility of $\alpha$-adrenoreceptor antagonists in patients with nephrolithiasis. They have previously been thought to reduce stone transit time and the frequency of pain episodes; ${ }^{8}$ the oral formulation and longer duration of action renders them ideal agents for long-term management and has rightly therefore made them a research focus in the past. However, their use in the emergency department for acute pain control is unfortunately limited by the slow onset time

\begin{tabular}{ll}
$\begin{array}{l}\text { Table } 1 \text { A table of articles excluded after review of the } \\
\text { full text }\end{array}$ \\
\begin{tabular}{ll} 
Full-text articles reviewed with rationale for exclusion \\
\hline Study & Reasons for exclusion \\
\hline Aronson and & Not a clinical trial; opinion piece re: \\
Hauben & adverse drug reactions \\
Erickson and $_{\text {Lieske }}^{27}$ & Not a clinical trial; topic review of renal \\
Helviz et af & calculi \\
& Not a clinical trial; case report of \\
Reed $^{29}$ & symmetrical peripheral gangrene \\
& Not a clinical trial; drug information re: \\
& hormone therapy
\end{tabular} \\
\hline
\end{tabular}

and a lack of evidence of any effect on acute pain; clinical trials have not been powered to detect effects on pain in the acute situation. Recently, significant doubts about their efficacy as expulsive therapies have been raised. ${ }^{30}$

Conversely, the potential advantages of $\beta$-agonists include their rapid onset time (almost immediate when administered either by the nebulised or intravenous route) and the pre-existing familiarity with their use that exists within acute settings. The potential for both systemic and topical action is also clearly advantageous. Despite some concerns raised within the literature, ${ }^{18}$ side effects are usually well tolerated ${ }^{31}$ (especially in the absence of significant comorbidity) and a patient group has suggested that the most common side effects (tremor, tachycardia and restlessness) are likely to be an acceptable trade-off in return for improved analgesia. (Tabner A. Patient and Public Involvement Meeting Summary—SARC, unpublished).

\section{CONCLUSION}

There is no evidence to support or refute the proposed use of $\beta$-agonists for analgesia in patients with renal colic.

Further research on this topic has been suggested as long ago as $1975 .^{32} \beta$-agonists are cheap, readily available and have predictable, manageable and relatively mild side effects. Given the clinical need, the clear biological plausibility, the animal model evidence and the limited but promising human studies (especially Jung et $a l^{19}$ ), 
clinical trials investigating the use of $\beta$-agonists in the acute setting for renal colic are recommended.

Twitter Follow Andrew Tabner at @andrewtabner

Acknowledgements The authors thank their colleagues at both the Royal College of Emergency Medicine Annual Scientific Conference 2014 (Dragon's Den event) and the Clinical Studies Group Meeting 2015 who recommended the conduct of this review.

Contributors AJT, GDJ and RIL conceived the study. AJT, GDJ, AF and JS planned the study. JS conducted the database searches. AJT and GDJ performed the hand searches, screened the records and reviewed the full-text articles. AJT and GDJ composed the first draft of the manuscript. All the authors revised the manuscript critically for important intellectual content, have given final approval of the version and agree to be accountable for all aspects of the work.

Funding This research received no specific grant from any funding agency in the public, commercial or not-for-profit sectors.

Competing interests The authors are part of the research group planning the 'Salbutamol for Analgesia in Renal Colic-SARC' trial.

Provenance and peer review Not commissioned; externally peer reviewed.

Data sharing statement No additional data are available.

Open Access This is an Open Access article distributed in accordance with the Creative Commons Attribution Non Commercial (CC BY-NC 4.0) license, which permits others to distribute, remix, adapt, build upon this work noncommercially, and license their derivative works on different terms, provided the original work is properly cited and the use is non-commercial. See: http:// creativecommons.org/licenses/by-nc/4.0/

\section{REFERENCES}

1. Curhan GC. Epidemiology of stone disease. Urol Clin North Am 2007;34:287-93.

2. Bultitude M, Rees J. Management of renal colic. BMJ 2012;345:e5499.

3. Davenport K, Waine $\mathrm{E}$. The role of non-steroidal anti-inflammatory drugs in renal colic. Pharmaceuticals 2010;3:1304-10.

4. Holdgate A, Pollock T. Nonsteroidal anti-inflammatory drugs (NSAIDs) versus opioids for acute renal colic. Cochrane Database Syst Rev 2004;(1):CD004137.

5. Electronic Medicines Compendium. Summary of product characteristics: Ventolin $0.5 \mathrm{mg}$ in $1 \mathrm{ml}$ solution for injection. (cited 23 January 2015). http://www.medicines.org.uk/emc/medicine/3637/SPC/

6. Joint Formulary Committee. British National Formulary (online). London: BMJ Group and Pharmaceutical Press, 2015. (cited 10 January 2015). http://www.medicinescomplete.com

7. Malin JMJ, Deane RF, Boyarsky S. Characterisation of adrenergic receptors in human ureter. Br J Urol 1970;42:171-4.

8. Campschroer T, Zhu Y, Duijvesz D, et al. Alpha-blockers as medical expulsive therapy for ureteral stones. Cochrane Database Syst Rev 2014;(4):CD008509.

9. Matsumoto R, Otsuka A, Suzuki T, et al. Expression and functional role of $\beta 3$-adrenoceptors in the human ureter. Int $J$ Urol 2013;20:1007-14

10. Mudraia IS, Kirpatovski VI. [Adrenergic regulation of the contractility of the human and canine ureters]. Biull Eksp Biol Med 1992;113:363-5.
11. Park YC, Tomiyama $\mathrm{Y}$, Hayakawa $\mathrm{K}$, et al. Existence of a beta3-adrenoceptro and its functional role in the human ureter. $J$ Urol 2000;164:1364-70.

12. Tomiyama $\mathrm{Y}$, Murakami M, Hayakawa $\mathrm{K}$, et al. Pharmacological profile of KUL-7211, a selective beta-adrenoceptor agonist, in isolated ureteral smooth muscle. J Pharmacol Sci 2003:92:411-19.

13. Jung HU, Frimodt-Møller PC, Osther PJ, et al. Pharmacological effect on pyeloureteric dynamics with a clinical perspective: a review of the literature. Urol Res 2006;34:341-50.

14. Rose JG, Gillenwater JY. The effect of adrenergic and cholinergic agents and their blockers upon ureteral activity. Invest Urol 1974:11:439-51.

15. McLeod DG, Reynolds DG, Swan KG. Adrenergic mechanisms in the canine ureter. Am J Physiol 1973;224:1054-8.

16. Kondo S, Morita T, Saeki H, et al. Effects of autonomic drugs on in vivo recording of electromyograms of canine renal pelvis and ureter. Urol Int 1985;40:260-6.

17. Morita T, Suzuki T, Kondo S, et al. Relationship between pelviureteral peristaltic frequency and urine flow change evoked by autonomic drug administration. Tohoku J Exp Med 1985; 146:265-71

18. Danuser H, Weiss R, Abel D, et al. Systemic and topical drug administration in the pig ureter: effect of phosphodiesterase inhibitors alpha1, beta and beta2-adrenergic receptor agonists and antagonists on the frequency and amplitude of ureteral contractions. $J$ Urol 2001;166:714-20.

19. Jung H, Nørby B, Frimodt-Møller PC, et al. Endoluminal isoproterenol irrigation decreases renal pelvic pressure during flexible ureterorenoscopy: a clinical randomized, controlled study. Eur Urol 2008;54:1404-13.

20. Wanajo I, Tomiyama Y, Tadachi M, et al. The potency of KUL-7211, a selective ureteral relaxant, in isolated canine ureter: comparison with various spasmolytics. Urol Res 2005;33:409-14.

21. Wanajo I, Tomiyama Y, Yamazaki Y, et al. Ureteral selectivity of intravenous $\beta$-adrenoceptor agonists in pig model of acute ureteral obstruction: comparison of KUL-7211, a selective $\beta 2 / \beta 3$ agonist, with isoproterenol, terbutaline, and CL-316243. Urology 2011;77:1266. e1-6.

22. Morgan DJ, Paull JD, Richmond BH, et al. Pharmacokinetics of intravenous and oral salbutamol and its sulphate conjugate. Br J Clin Pharmacol 1986;22:587-93.

23. National Institute for Health Research. UK Clinical Trials Gateway. https://www.crn.nihr.ac.uk/can-help/funders-academics/nihrcrnportfolio/ (accessed 17 Feb 2015)

24. National Institute for Health Research. Clinical Research Network Portfolio.

25. U.S. National Institutes of Health. ClinicalTrials.gov. https:// clinicaltrials.gov (accessed 17 Feb 2015).

26. Aronson JK, Hauben M. Anecdotes that provide definitive evidence. Br Med J 2006;333:1267-9.

27. Erickson SB, Lieske JC. Medical management. Clin Rev Bone Mine Metab 2004:2:237-51.

28. Helviz Y, Hersch M, Shmuelevitz L, et al. Bad to worse. Am J Med 2011;124:215-17.

29. Reed M. Outlook: clinical trials. Pharm Times 2010;76(12). http:// www.pharmacytimes.com/publications/issue/2010/december2010/ ClinicalTrials-1210 (accessed 17 Feb 2015).

30. Pickard R, Starr K, MacLennan G, et al. Medical expulsive therapy in adults with ureteric colic: a multicentre, randomised, placebo-controlled trial. Lancet 2015;386:341-9.

31. Price AH, Clissold SP. Salbutamol in the 1980s. A reappraisal of its clinical efficacy. Drugs 1989;38:77-122.

32. Peters HJ, Eckstein W. Possible pharmacological means of treating renal colic. Urol Res 1975;3:55-9. 